ORIGINAL ARTICLE

\section{Use of Vibrio cholerae Vaccine in an Outbreak in Guinea}

\author{
Francisco J. Luquero, M.D., M.P.H., Lise Grout, D.V.M., M.P.H., Iza Ciglenecki, M.D., \\ Keita Sakoba, M.D., Bala Traore, M.D., Melat Heile, N.P., Alpha Amadou Diallo, M.Sc., \\ Christian Itama, M.D., Anne-Laure Page, Ph.D., Marie-Laure Quilici, Ph.D., \\ Martin A. Mengel, M.D., Jose Maria Eiros, M.D., Ph.D., Micaela Serafini, M.D., M.P.H., \\ Dominique Legros, M.D., M.P.H., and Rebecca F. Grais, Ph.D.
}

\section{A BSTRACT}

\section{BACKGROUND}

The use of vaccines to prevent and control cholera is currently under debate. Shanchol is one of the two oral cholera vaccines prequalified by the World Health Organization; however, its effectiveness under field conditions and the protection it confers in the first months after administration remain unknown. The main objective of this study was to estimate the short-term effectiveness of two doses of Shanchol used as a part of the integrated response to a cholera outbreak in Africa.

\section{METHODS}

We conducted a matched case-control study in Guinea between May 20 and October 19, 2012. Suspected cholera cases were confirmed by means of a rapid test, and controls were selected among neighbors of the same age and sex as the case patients. The odds of vaccination were compared between case patients and controls in bivariate and adjusted conditional logistic-regression models. Vaccine effectiveness was calculated as $(1$-odds ratio $) \times 100$.

\section{RESULTS}

Between June 8 and October 19, 2012, we enrolled 40 case patients and 160 controls in the study for the primary analysis. After adjustment for potentially confounding variables, vaccination with two complete doses was associated with significant protection against cholera (effectiveness, 86.6\%; 95\% confidence interval, 56.7 to 95.8; $\mathrm{P}=0.001$ ).

\section{CONCLUSIONS}

In this study, Shanchol was effective when used in response to a cholera outbreak in Guinea. This study provides evidence supporting the addition of vaccination as part of the response to an outbreak. It also supports the ongoing efforts to establish a cholera vaccine stockpile for emergency use, which would enhance outbreak prevention and control strategies. (Funded by Médecins sans Frontières.)
From Epicentre (F.J.L., L.G., A.-L.P., R.F.G.), African Cholera Surveillance Network, Agence de Médicine Préventive (K.S., M.A.M.), and National Reference Center for Vibrios and Cholera, Enteric Bacterial Pathogens Research and Expertise Unit, Institut Pasteur (M.-L.Q.) - all in Paris; Médecins sans Frontières, Geneva (L.G., I.C., M.S., D.L.); Ministry of Health (K.S.), Direction Préfectorale de la Santé (B.T.) and Research and Documentation Service, Ministry of Health (A.A.D.), Médecins sans Frontières (M.H.), and World Health Organization (C.I.) - all in Conakry, Guinea; and the Department of Microbiology, University of Valladolid, Valladolid, Spain (J.M.E.). Address reprint requests to Dr. Luquero at Epicentre, 8 rue Saint Sabin, 75011 Paris, France, or at francisco.luquero@epicentre.msf.org.

N Engl J Med 2014;370:2111-20. DOI: 10.1056/NEJMoal312680

Copyright $\odot 2014$ Massachusetts Medical Society. 
ARGE-SCALE CHOLERA OUTBREAKS HAVE shown the limits of traditional response strategies. ${ }^{1}$ The devastating consequences of cholera epidemics in vulnerable populations have opened a debate about ways to improve preparedness and response plans. Two documents, issued by the World Health Organization (WHO) in 2010 and 2011, have also stimulated debate: a revised position paper regarding oral cholera vaccines $^{2}$ and the prequalification of the cholera vaccine Shanchol (Shantha Biotech). ${ }^{3}$

The two oral cholera vaccines currently prequalified by the WHO are killed whole-cell Vibrio cholerae serogroup 01 vaccines: Shanchol also contains $V$. cholerae serogroup O139, and Dukoral (Crucell) contains a recombinant cholera toxin B subunit. Dukoral was shown to provide $84 \%$ protection in the short term (6 months) under field conditions and has been used successfully in Asia and Africa. ${ }^{4,5}$ However, the effectiveness of Shanchol under field conditions needs to be determined, because the efficacy of the vaccine has been measured only under experimental conditions in Kolkata, India. ${ }^{6}$ Furthermore, because that trial was designed to evaluate protection in the long term (at 2, 3, and 5 years after vaccination), the protection offered by Shanchol within the first months after vaccination remains unknown. ${ }^{6-8}$

Shanchol differs from Dukoral in important ways: its price is lower ( $\$ 1.85$ vs. $\$ 5.25$ per $\operatorname{dose}^{9}$ ), it does not require buffer, and its storage volume is lower, ${ }^{10}$ which reduces the logistic burden during mass vaccination campaigns. Evidence about the protection conferred by Shanchol in the first months after administration under field conditions is essential for the consideration of its use in an outbreak. This is especially true at a time when the WHO and its partners are in the process of creating a stockpile of cholera vaccine for emergency use. ${ }^{11}$ In this study, we provide estimates of the effectiveness of Shanchol when used in response to an outbreak in an African country.

\section{METHODS}

\section{STUDY OVERSIGHT}

This study was sponsored by Médecins sans Frontières. The authors assume full responsibility for the analyses and interpretation of the data. The study protocol was approved by the ethics review boards of Guinea and Médecins sans Frontières. Vaccine and treatment were provided free of charge, and participation in the study was voluntary. Written informed consent was obtained from participants or their parents or guardians. Adverse events were documented by means of passive surveillance for 14 days after immunization, as described elsewhere. ${ }^{12}$

\section{STUDY SETTING}

The seventh cholera pandemic spread to Guinea in 1970; the largest outbreak to date was observed in 1994, with more than 30,000 cases and 670 deaths reported nationwide. From 2003 through 2007, cholera outbreaks were reported in the capital, Conakry, and in the coastal zones during the rainy season (July through September). A new cholera outbreak was declared in Guinea in February 2012 (see the Supplementary Appendix, available with the full text of this article at NEJM.org). The National Microbiology Laboratory in Conakry confirmed the circulation of $V$. cholerae serogroup 01 , biotype El Tor. Outbreak response interventions were implemented, including distribution of freeof-charge medical treatment, as well as efforts to improve access to safe water, improve sanitation, and promote hygiene. ${ }^{13-15}$ In addition, nonselective mass vaccination campaigns were implemented in the prefectures of Boffa and Forécariah (two doses of Shanchol with an interval of at least 2 weeks between doses) in accordance with WHO recommendations. ${ }^{2}$ In Boffa, residents of the six subprefectures bordering the ocean (Koba, BoffaCenter, Douprou, Tougnifili, and parts of Mankountan and Tamita) were vaccinated during the period from April 18 to May 14; in Forécariah, the residents of the subprefectures of Kaback and Kakossa were vaccinated during the period from May 27 to June 15 (Fig. S3 in the Supplementary Appendix). Details of the vaccination campaigns have been published elsewhere. ${ }^{12,16}$

\section{SURVEILLANCE FOR CHOLERA}

Between April 16 and October 31, 2012, the surveillance system was reinforced in 1 prefectural hospital, 8 health centers, and 23 health posts in the vaccinated areas. The case definition of suspected cholera (acute, nonbloody, watery diarrhea with more than three liquid stools in 24 hours) was standardized, and a case-based notification system with cholera-specific registers was implemented in collaboration with the African Cholera Surveillance Network (Africhol). The medical staff was also trained in the use of a rapid diagnostic test for cholera (Crystal VC, Span Diagnostics). 


\section{DEFINITION AND SELECTION OF CASE PATIENTS}

All patients with suspected cholera who were seeking care in a health center in the study area between 1 week after the end of the vaccination campaigns and October 31, 2012, were eligible to be included as case patients if they provided written informed consent and fulfilled the following criteria: they had resided in the study area since April 16, 2012; they were older than 12 months of age; they had a positive result of a rapid diagnostic test for cholera; and their residence could be located after discharge. Persons with repeated episodes of watery diarrhea during the study periods were included in the study only for the first episode.

To assess whether effectiveness estimates could be attributed to differences in health-seeking behavior between case patients and controls, case patients with noncholera diarrhea (i.e., those with a negative rapid diagnostic test result) were also compared with controls who did not have diarrhea (bias-indicator study). ${ }^{4}$

\section{DEFINITION AND SELECTION OF CONTROLS}

A neighbor of the same sex and within the same age group (1 to 4,5 to 9,10 to 19,20 to 29,30 to 39 , or 40 years of age or older) as the case patient was eligible to be a control if he or she had not sought treatment for diarrhea between January 1, 2012, and the date of onset of the matched case patient's diarrheal illness and if he or she would have sought treatment in a health center if severe, watery diarrhea had developed. Eligible controls were also required to meet the same informed consent and residency and age criteria that were used to select the case patients.

Four neighbor controls were selected for each case patient and were included in the primary analysis and the bias-indicator analysis (Fig. 1). Beginning with the first household to the left of the case patient's residence and using a sampling interval of three households, we recruited one control per household until four had been recruited.

\section{ASCERTAIN MENT OF VACCINATION}

\section{AND POTENTIALLY CONFOUNDING VARIABLES}

Receipt of the cholera vaccine during the mass immunization program was ascertained in faceto-face home interviews. Participants were asked whether they had been vaccinated and, if so, to show the vaccination cards. For those who reported that they had been vaccinated but were not in possession of a card, vaccination status and the completeness of dose ingestion were recorded as orally reported by the participant. Clinical, demographic, socioeconomic, and environmental variables were ascertained with the use of questionnaires.

\section{LABORATORY PROCEDURES}

For each case patient included in the study, a stool sample was collected and used to perform the rapid diagnostic test. The doctor or nurse in charge of the health center performed the test and interpreted the result in accordance with the manufacturer's instructions.

In addition, for patients with a positive rapid diagnostic test result, a filter-paper disk was dipped into fresh stool and placed in a microtube with two or three drops of normal saline solution ( $0.9 \%$ sodium chloride). Tubes were stored at room temperature and sent to Institut Pasteur (Paris) for isolation of $V$. cholerae with the use of standard methods. ${ }^{17}$ A polymerase chain-reaction (PCR) assay was systematically performed on all specimens. Detection of the $\mathrm{rfb}$ gene for the identification of $V$. cholerae was performed as described by Hoshino et al. ${ }^{18}$ PCR amplification of an exogenous internal positive control (TaqMan, Applied Biosystems) incorporated into each sample and amplification of the 16S ribosomal RNA (rRNA) gene were used to control for PCR inhibitors and bacterial DNA, respectively.

\section{STATISTICAL ANALYSIS}

In the primary analysis, we assessed the protection conferred by two completely ingested doses of vaccine against cholera confirmed by rapid diagnostic testing. Assuming 50\% vaccine effectiveness, we calculated that we would need to include 90 case patients and 360 controls (ratio, $1: 4)$ for the study to have $80 \%$ power at an alpha error of 5\%. In the secondary analysis, we assessed the protection conferred by an incomplete course of vaccination (one complete dose, or one or two doses that were incomplete because the patient spat out the dose or vomited). We also conducted a subanalysis in which case patients were considered to be those in whom the presence of $V$. cholerae was confirmed by culture, PCR, or both, as well as a bias-indicator analysis in which case patients were considered to be those who had diarrhea but with a negative result of rapid diagnostic testing.

We compared the odds of vaccination between case patients and controls through condi- 


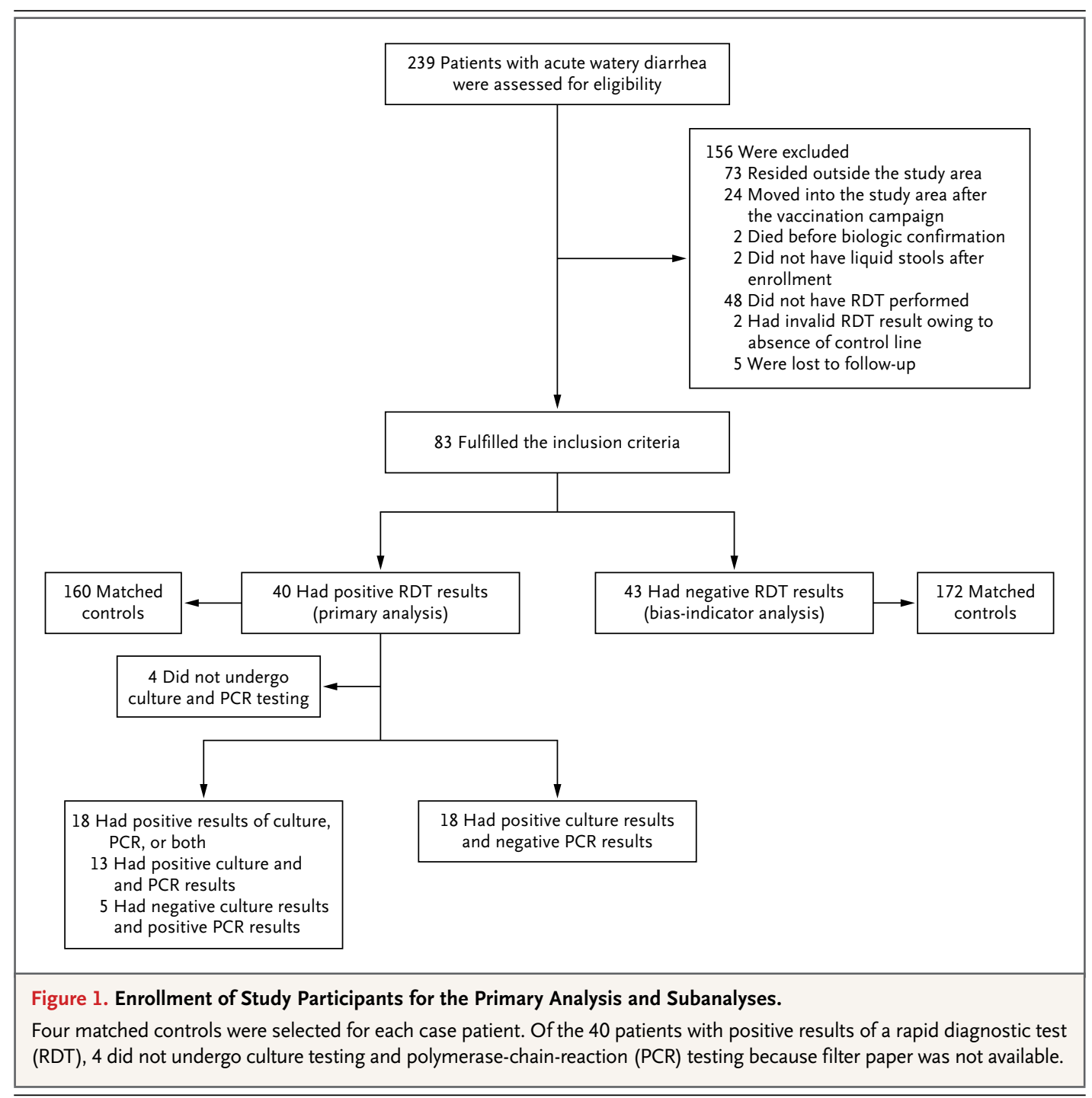

tional logistic regression to account for the matching design; we fit a model with indicator variables for nonvaccinated participants and participants who received incomplete or complete doses of vaccine. We calculated the level of vaccine protection as $(1$-odds ratio $) \times 100$.

We compared demographic, environmental, and socioeconomic factors between case patients and matched controls to assess their potential as confounders of vaccine protection. We considered as possible confounders variables for which $P$ values were less than 0.20 in the bivariate models. We obtained an adjusted estimate of vaccine protection by adjusting for covariables that significantly improved the likelihood of the model. All reported P values and 95\% confi- dence intervals are two-sided. A P value of less than 0.05 was considered to indicate statistical significance. Stata/SE, version 10 (StataCorp), was used for analyses.

\section{RESULTS}

\section{BASELINE CHARACTERISTICS}

From May 21 to October 31, 2012, a total of 239 patients with acute, nonbloody diarrhea were treated at health centers in the study area, 5 of whom died (case fatality rate, $2 \%$ ); 40 of the 239 patients and 160 controls were enrolled in the primary analysis between June 8 and October 19, 2012 (Fig. 1 and 2). None of the case patients enrolled in the study died. The median age of the 
study participants was 28.0 years (interquartile range, 16.5 to 39.0 ); $35 \%$ of the participants were female (Table 1). Half the case patients sought care on the day of symptom onset. At the time of admission, $70 \%$ of the case patients were dehydrated.

Of 36 case patients included in the primary analysis for whom a specimen was sent for culture and PCR analysis, 18 (50\%) were positive for $V$. cholerae serogroup $\mathrm{O} 1$ biotype $\mathrm{El}$ Tor, serotype Ogawa; 13 had positive results of culture and PCR, and 5 had positive PCR results but negative culture results. Samples from all 36 case patients showed a weak amplification signal for the 16S rRNA gene. Among the 18 negative specimens, 5 had an almost undetectable amplification signal.

In addition, 43 case patients with watery diarrhea and a negative rapid diagnostic test result and 172 controls were recruited for the bias-indicator analysis (Fig. 1 and 2).

\section{ANALYSIS OF CONFOUNDERS AND EFFECT MODIFIERS}

Case patients with cholera confirmed by rapid diagnostic testing were more likely than controls to have eaten in public places and to have shared a latrine with persons who had cholera (Table 1, and Table S1 in the Supplementary Appendix). The baseline characteristics of the case patients without cholera and the matched controls included in the bias-indicator analysis are shown in Table S2 in the Supplementary Appendix.

\section{VACCINE EFFECTIVENESS}

In the crude analysis and after adjustment for potential confounders, vaccination with two complete doses was associated with significant protection against cholera (adjusted effectiveness, $86.6 \%$; $95 \%$ confidence interval [CI], 56.7 to $95.8 ; \mathrm{P}=0.001$ ) (Table 2). The estimate of the effectiveness of an incomplete course of vaccine was not precise enough to be conclusive $(42.8 \%$; 95\% CI, -83.6 to $82.2 ; \mathrm{P}=0.35$ ).

In the subanalysis that included only case patients with cholera confirmed by culture, PCR, or both, vaccination with two complete doses was also associated with significant protection against cholera $(91.6 \%$; $95 \%$ CI, 58.6 to 98.3 ; $\mathrm{P}=0.002$ ) (Table 3). The odds of vaccination did not differ significantly between patients with watery diarrhea not caused by cholera and controls, suggesting the absence of bias in the primary analysis (Table 3).

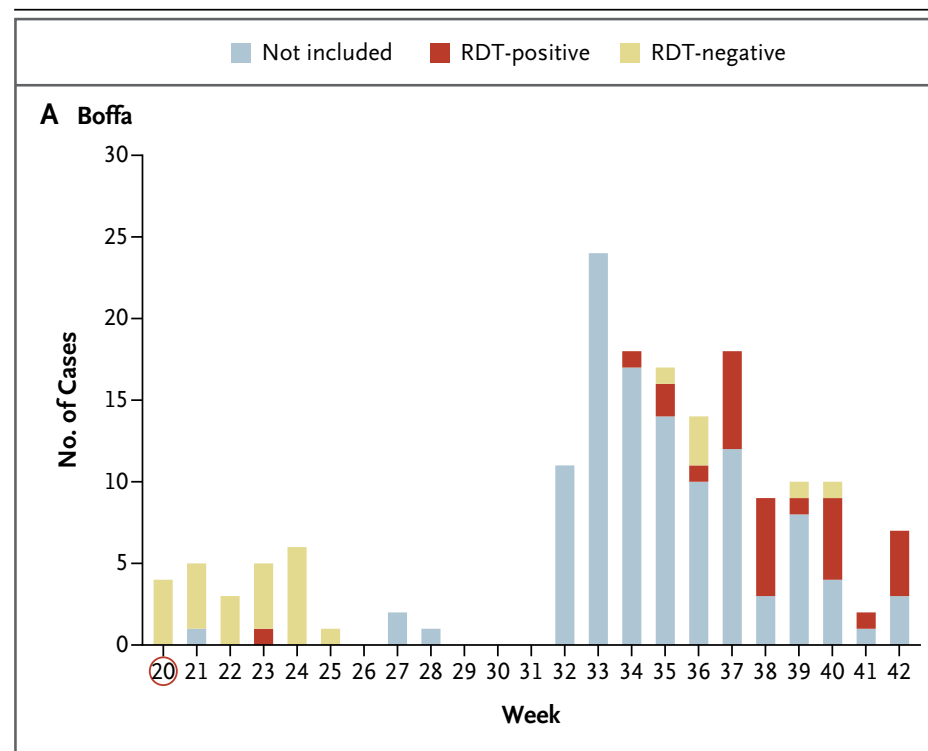

B Forécariah

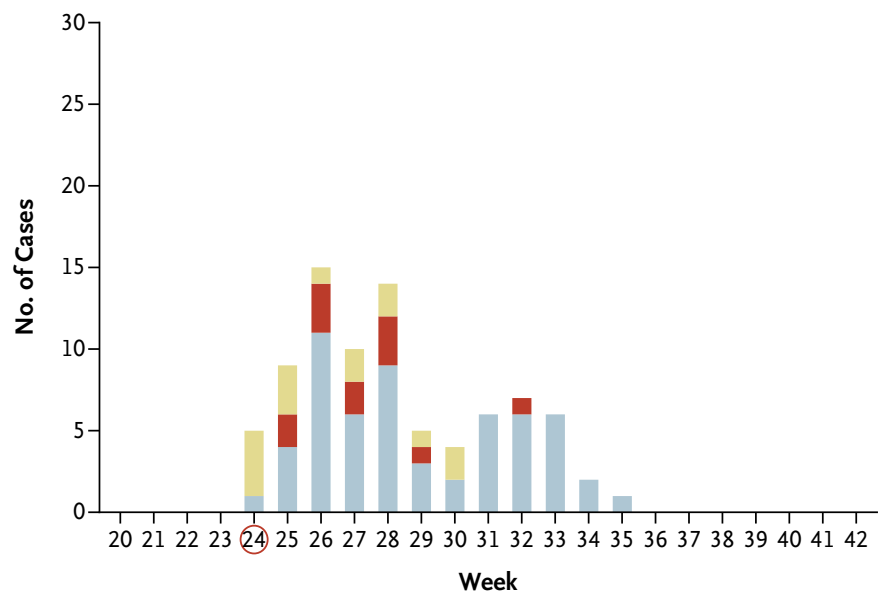

Figure 2. Cases of Acute Diarrhea Reported in the Study Areas.

Cases reported after the starting date of the study (circled in red; week 20 of the calendar year in the Boffa prefecture and week 24 in the Forécariah prefecture) are shown. The cholera cases confirmed by RDT and included in the primary analysis are shown in red, the noncholera cases (RDT-negative) included in the bias-indicator analysis are shown in yellow, and suspected cholera cases not included in the analysis are shown in gray.

\section{DISCUSSION}

Our results show the effectiveness of two complete doses of Shanchol administered as part of the response to a cholera epidemic in Africa. Our estimate of the short-term protection conferred by Shanchol is in line with previous results for Dukoral. ${ }^{4}$ This is relevant in light of the low levels of protection provided by oral vaccines in lowincome African settings in the past. ${ }^{19-21}$ 
This study was performed under field conditions during a cholera outbreak and therefore has several limitations. The immunization campaign in response to the outbreak was part of the control strategy implemented by the Guinea Ministry of Health with the support of Médecins sans Frontières; thus, exposure to the vaccine in the population was not controlled. Overall, 316,250 vaccines were delivered, and 48 patients reported nonsevere adverse events after immunization (Table S4 in the Supplementary Appendix); the vaccine coverage ranged from $69 \%$ in the Koba subprefecture to $84 \%$ in the Mankountan subprefecture. ${ }^{12}$ High vaccination coverage reduces transmission in vaccinated communities (herd protection), ${ }^{22-24}$ thereby directly and indirectly reducing the risk of cholera. In the past, this has limited opportunities to measure vaccine effectiveness ${ }^{25}$ because of difficulties in recruiting case patients. In our study, most of the case patients were recruited from Koba (in the Boffa prefecture), where a small local outbreak was reported (August through October 2012). Koba had the lowest vaccination coverage in the study area, ${ }^{12}$ and it borders Dubreka, where high transmission was reported (overall attack rate, 17 cases per 10,000 population) (see the Supplementary Appendix). Although the sample was small, the power was high (99\%), because the observed vaccine effectiveness was higher than the value assumed for the calculation of sample size. However, the small sample limited the possibility of conducting analyses stratified by age and of estimating the effectiveness of a single dose with sufficient precision. This latter estimate of effectiveness, although not significant,

\begin{tabular}{|c|c|c|c|}
\hline Characteristic & $\begin{array}{l}\text { Controls } \\
(N=160)\end{array}$ & $\begin{array}{l}\text { Case Patients } \\
\qquad(\mathrm{N}=40)\end{array}$ & $\mathrm{P}$ Value \\
\hline Female sex - no. (\%) & $56(35)$ & $14(35)$ & \\
\hline \multicolumn{4}{|l|}{ Age $-y r$} \\
\hline Median & 28 & 28 & \\
\hline Interquartile range & $16-39$ & $18-36$ & \\
\hline Occupation - no. (\%) & & & 0.18 \\
\hline Trader & $29(18)$ & $8(20)$ & \\
\hline Farmer & $37(23)$ & $16(40)$ & \\
\hline Student & $29(18)$ & $3(8)$ & \\
\hline Fisherman & $10(6)$ & $3(8)$ & \\
\hline Housewife & $10(6)$ & $1(3)$ & \\
\hline Unemployed & $22(14)$ & $6(15)$ & \\
\hline Other & $23(14)$ & $3(8)$ & \\
\hline Educational level and literacy of head of household — no./total no. (\%) & & & 0.13 \\
\hline None & $43 / 158(27)$ & $13 / 40(33)$ & \\
\hline Primary school & $5 / 158(3)$ & $4 / 40(10)$ & \\
\hline Secondary school & $21 / 158(13)$ & $2 / 40(5)$ & \\
\hline University & $5 / 158(3)$ & 0 & \\
\hline Literate & $84 / 158(53)$ & $21 / 40(53)$ & \\
\hline Has telephone in home - no. (\%) & $128(80)$ & $27(68)$ & 0.10 \\
\hline Household size - no. (\%) & & & 0.06 \\
\hline 0-4 members & $34(21)$ & $17(43)$ & \\
\hline $5-7$ members & $40(25)$ & $7(18)$ & \\
\hline $8-12$ members & $49(31)$ & $9(23)$ & \\
\hline >12 members & $37(23)$ & $7(18)$ & \\
\hline
\end{tabular}




\begin{tabular}{|c|c|c|c|}
\hline Characteristic & $\begin{array}{l}\text { Controls } \\
(\mathrm{N}=160)\end{array}$ & $\begin{array}{l}\text { Case Patients } \\
(N=40)\end{array}$ & P Value \\
\hline $\begin{array}{l}\text { Proportion of children in the household attending school } \\
\qquad \text { - no./total no. (\%) }\end{array}$ & & & 0.13 \\
\hline None & $33 / 144(23)$ & $14 / 37(38)$ & \\
\hline Less than half & $42 / 144(29)$ & $11 / 37(30)$ & \\
\hline Half or more & $51 / 144(35)$ & $8 / 37(22)$ & \\
\hline All & $18 / 144(13)$ & $4 / 37(11)$ & \\
\hline Distance to the closest health center - no. (\%) & & & 0.10 \\
\hline Transportation required & $107(67)$ & $31(78)$ & \\
\hline Walking distance & $53(33)$ & $9(23)$ & \\
\hline $\begin{array}{l}\text { Lives in household with one or more other persons with cholera } \\
\qquad \text { - no./total no. (\%) }\end{array}$ & $4 / 159(3)$ & $3 / 40(8)$ & 0.15 \\
\hline Drinking water treated in the previous week — no. (\%) $\dagger$ & & & 0.15 \\
\hline No & $26(16)$ & $11(28)$ & \\
\hline At least 1 day & $34(21)$ & $5(13)$ & \\
\hline Every day & $100(63)$ & $24(60)$ & \\
\hline Ate food in a public place in the previous week - no./total no. (\%) & & & 0.02 \\
\hline Never & $72 / 160(45)$ & $11 / 39(28)$ & \\
\hline At least 1 day & $49 / 160(31)$ & $20 / 39(51)$ & \\
\hline Every day & $39 / 160(24)$ & $8 / 39(21)$ & \\
\hline Usual place of defecation — no. (\%) & & & 0.12 \\
\hline Latrine & $81(51)$ & $17(43)$ & \\
\hline Pit in the yard & $56(35)$ & $14(35)$ & \\
\hline On the ground & $23(14)$ & $9(23)$ & \\
\hline Shares a latrine with someone who has cholera - no./total no. (\%) & $5 / 136(4)$ & $6 / 30(20)$ & 0.001 \\
\hline
\end{tabular}

was substantially lower than that for two doses, and the point estimate was in line with previous findings with other oral cholera vaccines. ${ }^{4,6,24}$

Despite the wide and systematic distribution of vaccination cards and the short time span between the vaccination campaign and the data collection, $25 \%$ of the vaccinated people interviewed were not able to provide their vaccination card at the time of the study. Because we were not able to confirm vaccination status for all participants, some information bias may be present. Nevertheless, even if we consider the worstcase scenario, in which all the participants who were unable to find their vaccination card were not vaccinated, the vaccine effectiveness would still reach $82 \%$ (see the Supplementary Appendix).
Case-control studies of vaccine effectiveness may also be prone to bias related to differences in health-seeking behavior. To measure this potential bias, we conducted a supplementary, biasindicator analysis, comparing the odds of vaccination among patients with watery diarrhea not caused by cholera and a sample of matched controls. The odds of vaccination did not differ significantly between these two groups, and we interpret this finding as indicating the absence of a large bias in health-seeking behavior.

Despite the difficulties inherent in assessing vaccine effectiveness under field conditions, we were able to provide estimates of the short-term protection (during the first 6 months) conferred by Shanchol in Africa, where the effect of the 
Table 2. Effectiveness of Complete and Incomplete Vaccination.

\begin{tabular}{|c|c|c|c|c|c|c|}
\hline \multirow[t]{2}{*}{ Vaccination Status } & $\begin{array}{l}\text { Controls } \\
(\mathrm{N}=160)\end{array}$ & $\begin{array}{c}\text { Case } \\
\text { Patients } \\
(\mathrm{N}=40)\end{array}$ & $\begin{array}{l}\text { Crude Estimate } \\
\text { of Vaccine } \\
\text { Effectiveness* }\end{array}$ & $P$ Value & 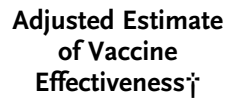 & P Value \\
\hline & \multicolumn{2}{|c|}{ no. of patients (\%) } & $\%(95 \% \mathrm{Cl})$ & & $\%(95 \% \mathrm{Cl})$ & \\
\hline No vaccination & $23(14)$ & $15(38)$ & Reference & & Reference & \\
\hline Incomplete vaccination $\Varangle$ & $36(23)$ & $14(35)$ & $38.9(-55.2$ to 76.0$)$ & 0.30 & 42.8 (-83.6 to 82.2$)$ & 0.35 \\
\hline Full vaccination & $101(63)$ & $11(28)$ & 84.0 (59.7 to 93.6$)$ & $<0.001$ & 86.6 (56.7 to 95.8$)$ & 0.001 \\
\hline
\end{tabular}

* Vaccine effectiveness was based on the odds of vaccination among case patients and controls; crude vaccine effectiveness was calculated as $(1$-odds ratio) $\times 100$.

$\dagger$ Estimates were adjusted according to the number of people living in the household, whether drinking water was treated, and whether a latrine was shared with a person who had cholera.

$¥$ Vaccination was considered incomplete if only one dose was taken or if one or both doses were spat out or vomited.

\begin{tabular}{|c|c|c|c|c|}
\hline \multirow[t]{2}{*}{ Subanalysis } & Controls & Case Patients & $\begin{array}{l}\text { Crude Estimate of } \\
\text { Vaccine Effectiveness }\end{array}$ & $P$ Value \\
\hline & \multicolumn{2}{|c|}{ no./total no. (\%) } & $\%(95 \% \mathrm{Cl})$ & \\
\hline \multicolumn{5}{|c|}{ Culture-PCR assay subanalysis $\uparrow$} \\
\hline No vaccination & $10 / 72(14)$ & $8 / 18(44)$ & Reference & \\
\hline Incomplete vaccination & $17 / 72(24)$ & $6 / 18(33)$ & $66.2(-53.0$ to 92.6$)$ & 0.16 \\
\hline \multirow[t]{3}{*}{ Full vaccination } & $45 / 72(63)$ & $4 / 18(22)$ & 91.6 (58.6 to 98.3 ) & 0.002 \\
\hline & Controls & $\begin{array}{l}\text { Patients with Diarrhea } \\
\text { Not Due to Cholera }\end{array}$ & $\begin{array}{c}\text { Crude Estimate of } \\
\text { Vaccine Effectiveness }\end{array}$ & P Value \\
\hline & \multicolumn{2}{|c|}{ no./total no. (\%) } & $\%(95 \% \mathrm{Cl})$ & \\
\hline \multicolumn{5}{|l|}{ Bias-indicator subanalysist } \\
\hline No vaccination & $9 / 172(5)$ & $4 / 43(9)$ & Reference & \\
\hline Incomplete vaccination & $35 / 172(20)$ & $7 / 43(16)$ & $48.1(-177.1$ to 90.3$)$ & 0.44 \\
\hline Full vaccination & $128 / 172(74)$ & $32 / 43(74)$ & $25.2(-225.2$ to 82.8$)$ & 0.70 \\
\hline
\end{tabular}

oral cholera vaccine is expected to be the highest in reducing mortality ${ }^{1,26}$ and where problems with the protection provided by oral vaccines have been documented in the past. ${ }^{21}$ The crude and adjusted estimates of effectiveness were similar after the effect of a large number of well-described possible confounders was explored, as well as when only cases confirmed by PCR or culture were considered. The low proportion of PCR-positive samples could be related to the small amount or the poor quality of biologic material, as indicated by the weak amplification signal of the
16S rRNA gene. False positive rapid diagnostic test results cannot be ruled out ${ }^{27,28}$; however, this nondifferential misclassification would have underestimated vaccine effectiveness.

The results presented here suggest that the protection conferred by Shanchol may be higher in the first 6 months than in the remaining 2 years after vaccination (an estimate of $67 \%$ was reported by Sur et al. for the 2-year period after vaccination $^{6}$ ), probably as a result of waning immunity. This difference may also be explained in part by the possibility that some nonvaccinated 
participants in the study by Sur et al. had naturally acquired immunity because cholera is endemic in Kolkata. In addition, our estimate might reflect some indirect protection, although indirect effects were minimized through the matched design. ${ }^{22,23}$

A factor that does not seem to have reduced the short-term protection provided by Shanchol is the cold-chain strategy used in Guinea, where vaccines were stored under cold chain but were transported and used at ambient temperature on the day of vaccination. The vial temperature monitor of each vaccine was checked for stability before administration, and all monitors indicated that the vaccines remained valid for use. These results are not surprising, in light of the good heat stability of Dukoral, ${ }^{29}$ but more robust documentation is required to allow for more flexible delivery strategies in the future. The use of oral cholera vaccine in outbreak contexts could also be substantially simplified if it were effective as a single-dose regimen. Our study was underpowered to provide precise estimates of the protection provided by a single dose of vaccine. Determining the short-term protection provided by one dose is a clear priority for the implementation of efficient and timely vaccination campaigns in response to cholera outbreaks.

In conclusion, our estimates of the shortterm effectiveness of Shanchol provide essential information regarding the rapid use of oral cholera vaccine to improve the current strategies for outbreak prevention and control. This evidence should serve to support the addition of oral cholera vaccines to the tools used in responses to epidemics.

Supported by Médecins sans Frontières.

Disclosure forms provided by the authors are available with the full text of this article at NEJM.org.

We thank the people of Guinea for their support and participation in the conduct of this survey; the Division of Disease Prevention and Control of the Ministry of Health of Guinea, Africhol, and all the district health and medical officers involved in the study for their support; Jean Rauzier for the work performed at the French National Reference Center for Vibrios and Cholera, which is funded by the Institut Pasteur and the French Institute for Public Health Surveillance; Charles Gaudry, head of mission for Médecins sans Frontières in Guinea, and the emergency coordinators, logisticians, and administrative staff of the Médecins sans Frontières Geneva operational center for their patience and enthusiastic support; the study teams and the Médecins sans Frontières teams in Boffa and Forécariah, especially the research assistants Drs. Soumah Abubacar and Oumar Touré; and Dr. Pedro Pablo Palma, medical director of the Médecins sans Frontières Barcelona-Athens operational center, for his comments and insight into this project and his continuing support.
REFERENCES

1. Ali M, Lopez AL, You YA, et al. The global burden of cholera. Bull World Health Organ 2012;90:209A-218A.

2. Cholera vaccines: WHO position paper. Wkly Epidemiol Rec 2010;85:117-28.

3. Harris JB, LaRocque RC, Qadri F, Ryan ET, Calderwood SB. Cholera. Lancet 2012;379:2466-76.

4. Lucas MES, Deen JL, von Seidlein L, et al. Effectiveness of mass oral cholera vaccination in Beira, Mozambique. N Engl J Med 2005;352:757-67.

5. Clemens JD, Sack DA, Harris JR, et al. Field trial of oral cholera vaccines in Bangladesh. Lancet 1986;2:124-7.

6. Sur D, Lopez AL, Kanungo S, et al Efficacy and safety of a modified killedwhole-cell oral cholera vaccine in India: an interim analysis of a cluster-randomised, double-blind, placebo-controlled trial. Lancet 2009;374:1694-702. [Erratum, Lancet 2010;376:1392.]

7. Sur D, Kanungo S, Sah B, et al. Efficacy of a low-cost, inactivated whole-cell oral cholera vaccine: results from 3 years of follow-up of a randomized, controlled trial. PLoS Negl Trop Dis 2011;5(10): e1289.

8. Bhattacharya SK, Sur D, Ali M, et al. 5 Year efficacy of a bivalent killed whole-cell oral cholera vaccine in Kolkata, India: a cluster-randomised, double-blind, placebocontrolled trial. Lancet Infect Dis 2013;13: 1050-6. [Erratum, Lancet Infect Dis 2013; 13:1011.]

9. Shin S, Desai SN, Sah BK, Clemens JD. Oral vaccines against cholera. Clin Infect Dis 2011;52:1343-9.

10. Pastor M, Pedraz JL, Esquisabel A. The state-of-the-art of approved and underdevelopment cholera vaccines. Vaccine 2013; 31:4069-78.

11. Martin S, Costa A, Perea W. Stockpiling oral cholera vaccine. Bull World Health Organ 2012;90:714.

12. Luquero FJ, Grout L, Ciglenecki I, et al. First outbreak response using an oral cholera vaccine in Africa: vaccine coverage, acceptability and surveillance of adverse events, Guinea, 2012. PLoS Negl Trop Dis 2013;7(10):e2465.

13. Médecins Sans Frontières. Cholera guidelines. Paris: Médecins San Frontières, 2004.

14. First steps for managing an outbreak of acute diarrhoea. Geneva: World Health Organization, 2010 (http://www.who.int/ cholera/publications/firststeps/en/index .html).

15. Cholera outbreak: assessing the out- break response and improving preparedness. Geneva: World Health Organization, 2010 (http://www.who.int/cholera/ publications/OutbreakAssessment/en/ index.html).

16. Ciglenecki I, Sakoba K, Luquero FJ, et al. Feasibility of mass vaccination campaign with oral cholera vaccines in response to an outbreak in Guinea. PLoS Med 2013;10(9):e1001512.

17. Dodin A, Fournier JM. Diagnosis of the cholera vibrio Vibrio cholerae $\mathrm{O}_{1}$. In: Laboratory methods for the diagnosis of cholera vibrio and other vibrios. Paris: Institut Pasteur, 1992:59-82.

18. Hoshino K, Yamasaki S, Mukhopadhyay AK, et al. Development and evaluation of a multiplex PCR assay for rapid detection of toxigenic Vibrio cholerae O1 and O139. FEMS Immunol Med Microbiol 1998;20:201-7.

19. Madhi SA, Cunliffe NA, Steele D, et al. Effect of human rotavirus vaccine on severe diarrhea in African infants. N Engl J Med 2010;362:289-98.

20. Armah GE, Sow SO, Breiman RF, et al. Efficacy of pentavalent rotavirus vaccine against severe rotavirus gastroenteritis in infants in developing countries in subSaharan Africa: a randomised, double- 
blind, placebo-controlled trial. Lancet 2010; 376:606-14.

21. Holmgren J, Svennerholm A-M. Vaccines against mucosal infections. Curr Opin Immunol 2012;24:343-53.

22. Ali M, Emch M, von Seidlein L, et al. Herd immunity conferred by killed oral cholera vaccines in Bangladesh: a reanalysis. Lancet 2005;366:44-9.

23. Ali M, Sur D, You YA, et al. Herd protection by a bivalent killed whole-cell oral cholera vaccine in the slums of Kolkata, India. Clin Infect Dis 2013;56:112331.

24. Khatib AM, Ali M, von Seidlein L, et al. Effectiveness of an oral cholera vac- cine in Zanzibar: findings from a mass vaccination campaign and observational cohort study. Lancet Infect Dis 2012;12: 837-44.

25. Dorlencourt F, Legros D, Paquet C, Neira M, Ivanoff B, Le Saout E. Effectiveness of mass vaccination with $\mathrm{WC} / \mathrm{rBS}$ cholera vaccine during an epidemic in Adjumani district, Uganda. Bull World Health Organ 1999;77:949-50.

26. Zuckerman JN, Rombo L, Fisch A. The true burden and risk of cholera: implications for prevention and control. Lancet Infect Dis 2007;7:521-30.

27. Page A-L, Alberti KP, Mondonge V, Rauzier J, Quilici M-L, Guerin PJ. Evalua- tion of a rapid test for the diagnosis of cholera in the absence of a gold standard. PLoS One 2012;7(5):e37360.

28. Martinez-Pino I, Luquero FJ, Sakoba $\mathrm{K}$, et al. Use of a cholera rapid diagnostic test during a mass vaccination campaign in response to an epidemic in Guinea, 2012. PLoS Negl Trop Dis 2013;7(8): e2366.

29. Ahmed ZU, Hoque MM, Rahman AS, Sack RB. Thermal stability of an oral killed-cholera-whole-cell vaccine containing recombinant B-subunit of cholera toxin. Microbiol Immunol 1994;38:83742.

Copyright (C) 2014 Massachusetts Medical Society. 